\title{
Impact of lifestyle training on serum lipids of children and adolescents with dyslipidemia: a quasi- experimental study
}

\author{
Ali Yaghoobi ${ }^{1}$, Fatemeh Taheri ${ }^{2}$, Marjan Farzad ${ }^{3}$, Toba Kazemi ${ }^{4}$
}

\begin{abstract}
${ }^{1}$ MD, Student Research Committee, Medical School, Birjand University of Medical Sciences, Birjand, Iran
${ }^{2}$ MD, Pediatrician, Professor, Cardiovascular Diseases Research Center, Faculty of Medicine, Birjand University of Medical Sciences, Birjand, Iran

${ }^{3}$ M.Sc. of Nursing, Faculty Member, Cardiovascular Diseases Research Center, School of Nursing and Midwifery, Birjand University of Medical Sciences, Birjand, Iran

${ }^{4} \mathrm{MD}$, Cardiologist, Professor, Cardiovascular Diseases Research Center, Faculty of Medicine, Birjand University of Medical Sciences, Birjand, Iran
\end{abstract}

Type of article: Original

\begin{abstract}
Background: Dyslipidemia is one of the most important risk factors for cardiovascular diseases. It can cause a rise in the incidence of these diseases in adulthood. Lifestyle modification is a determinant factor for incidence, prognosis and complications of cardiovascular diseases.

Objective: The aim of the study was to evaluate the effect of lifestyle modification on serum lipids of children and adolescents with dyslipidemia.

Methods: This quasi-experimental study (before - and after design) was conducted on all dyslipidemia children and adolescents with dyslipidemia referred to the cardiovascular diseases research center of Birjand University of Medical Sciences (Iran) in 2015. Participants underwent 6 sessions of training, appropriate to their age, including fat definition and healthy lifestyle. Before the study and 6 months after trainings, in the same circumstances, subjects were examined for level of blood lipids and blood pressure. Data were analyzed using paired-samples ttest, Pearson correlation coefficient, and independent-samples t-test. P-value of less than 0.05 was considered statistically significant.

Results: This study was performed on 50 children with dyslipidemia, of whom $52 \%$ were female and $48 \%$ were male. The mean age of participants was $12.77 \pm 1.84$ years. The study showed a significant difference between the mean of cholesterol $(\mathrm{p}<0.001)$ and LDL $(\mathrm{p}=0.008)$ before and after the study. Comparing the changes made in the mean of variables such as FBS, Cholesterol, TG, HDL and LDL as a result of gender-based lifestyle modification, no significant difference between the two sexes was seen $(\mathrm{p}>0.05)$. Pearson correlation coefficient showed no significant relationship between the mean of these variables in children as a result of lifestyle modification and their age $(\mathrm{p}>0.05)$.

Conclusion: Lifestyle modification relying on exercise and physical activity, nutrition and sufficient sleep and rest can be effective as a non-pharmacological supplement in reducing blood lipids and dyslipidemia. Lifestyle modification training is essential for both children and their parents after diagnosis of dyslipidemia as a part of the Therapeutic plan.

Keywords: Dyslipidemia, Childhood, Lifestyle
\end{abstract}

\section{Introduction}

Non-communicable diseases are prevalent worldwide these days due to the development of technology, modernization and factors such as population growth and lifestyle changes. While imposing a high cost on

\section{Corresponding author:}

Professor Dr. Fatemeh Taheri, Cardiovascular Diseases Research Center, Faculty of Medicine, Birjand University of Medical Sciences, Birjand, Iran. Tel: +98.5632443000, Fax:+98.5632442088, Email: ftaheri64@yahoo.com

Received: February 17, 2017, Accepted: June 28, 2018, Published: November 2018

iThenticate screening: June 20, 2018, English editing: October 06, 2018, Quality control: October 08, 2018

This article has been reviewed / commented by three experts

Funding / research project approval: Grant Number 743, November 2015 (Birjand University of Medical Sciences)

Ethics approval: IR.BUMS.1394.8 (Birjand University of Medical Sciences)

(C) 2018 The Authors. This is an open access article under the terms of the Creative Commons Attribution-NonCommercialNoDerivs License, which permits use and distribution in any medium, provided the original work is properly cited, the use is non-commercial and no modifications or adaptations are made. 
healthcare systems, these diseases cause disability and reduce quality of life. Cardiovascular diseases, including atherosclerosis, are the most common non-communicable diseases in the world. It is predicted that these diseases will remain the most important cause of mortality (36\% of all deaths) worldwide by 2020 (1). Annually, 17 million people die of atherosclerosis due to coronary heart diseases, and these diseases account for 10 million deaths from 40 million deaths that occur annually in developing countries (2). Atherosclerosis is the leading cause of death in developed countries and is expected to be the main cause in developing countries within the next 25 years (3). The mortality rate of atherosclerotic heart diseases has steadily risen since 1990 and as the world's leading cause of mortality, was responsible for $84.5 \%$ of deaths reported in 2013. Disability Adjusted Life Years (DALYs) are also rising due to these diseases (4). According to studies conducted in Iran, the prevalence of the diseases in males and females was 1,436 and 1,168 per 100,000, respectively and the mortality rate of them is reported $31.8 \%(5)$.

Primary lesion in atherosclerosis occurs in intima and appears from the time of childhood through the formation of fatty streaks (6). It is now known that although symptoms of the disease appear in adulthood, the disease develops at childhood (7). Thus, the pathological changes in atherosclerosis begin from childhood and develop in several stages, and occur at older ages (8). The risk factors for atherosclerosis based on theepidemiological studies in children have been reported as obesity, lipid metabolism disorders (dyslipidemia), endothelial dysfunction, inflammatory factors, high blood pressure and physical activity (9-11). According to Skybo and Venger (12), the level of blood lipids have been reported as factors influencing the multifactorial atherosclerosis. High levels of saturated fats, which increase the fatty tissues, raise the risk of heart diseases due to the atherogenic lipoprotein enhancement (12). Several other studies have indicated the role of lipids and its concentration in atherosclerotic lesions $(3,6,13)$. Dyslipidemia, as one of the most important factors, leads to an increase in the blood serum level of one or a number of lipids such as cholesterol, triglycerides as Very Low Density Lipoprotein (VLDL) and Low Density Lipoprotein (LDL). These serum fats are often steady from childhood to puberty, although this trend is not absolute and the percentage of children who are initially in the high-risk group may gradually proceed into the middle risk group as they get older (14). According to numerous studies in Iran and other parts of the world, dyslipidemia in children and adolescents is prevalent (15-19). Considering that childhood dyslipidemia leads to early onset of atherosclerosis in adulthood, the target group for intervention and prevention of cardiovascular diseases are children and adolescents (20-21). Dyslipidemia modification and changes in blood lipid profiles due to the use of lipid lowering drugs, simultaneously, reduce the risk of mortality from cardiovascular diseases (22). In addition to drugs, lifestyle modification, systemic exercises and healthy diet can also have significant effects on dyslipidemia, especially in childhood (23). Lifestyle, as an important factor, has always been the focus of health education and promotion which consists of a high percentage (53\%) of the factors affecting health compared to other factors such as inheritance (16\%), health care (10\%) and environment (21\%) (24). Several studies have been conducted in Iran and worldwide on lifestyle, serum lipids, and cardiovascular diseases. Jalali et al. showed that inappropriate dietary pattern and low level of physical activities could be effective in increasing the level of blood lipids (25). Lack of observance of a healthy lifestyle as the main cause of dyslipidemia and progression of cardiovascular diseases has been reported by Kelishadi et al. (26). According to other studies, lifestyle modification and physical activity reduce blood lipids and the risk of cardiovascular diseases (27). As stated in the results of the studies and considering how lifestyle (type of diet, physical activity, etc.) is responsible for the occurrence of many non-communicable diseases, including cardiovascular disease lifestyle modification is of particular importance (28). In this regard, Lim et al. (2013) showed that low level of knowledge and undesirable physical activity are the two factors influencing lifestyle, and educational interventions such as educational programs can be effective in promoting lifestyle and reducing cardiovascular risk factors (29). Public awareness as one of the pillars of prevention programs is recommended in another study (30).

Considering that childhood and adolescent dyslipidemia, while having a destructive effect in these age groups, can lead to dangerous and irreversible complications in adulthood, it seems that lifestyle modification can be the main key to this problem. The aim of the study was to evaluate the effect of lifestyle modification on serum lipids of children and adolescents with dyslipidemia.

\section{Material and Methods}

\subsection{Research design and setting}

In this quasi-experimental, before - and after design study, all the children and adolescents referred to the Cardiovascular Diseases Research Center at Birjand University of Medical Sciences, (Iran) in 2015, and had dyslipidemia, entered the study. The reasons we chose that location are that the Cardiovascular Diseases Research Center is the only center for research on cardiovascular diseases in southern Khorasan, and it is also a referral center. 
The study included all children and adolescents with Dyslipidemia in Birjand who were selected from the previous research project (31).

\subsection{Selection criteria}

\subsubsection{Inclusion criteria}

The following were set as the inclusion criteria: 1) Age category of 6-18 years old, 2) Not having any history of high blood lipids in relatives of grade 1 and 2, genetically, 3) Not being treated with any special diet, 4) No use of any drugs that lower blood lipids and weight, 5) Presence of at least one disorder in fat profiles (Total Cholesterol $>200$ $\mathrm{mg} / \mathrm{dl}$, LDL-C $>130 \mathrm{mg} / \mathrm{dl}$, HDL-C $<40 \mathrm{mg} / \mathrm{dl}, \mathrm{TG}>130 \mathrm{mg} / \mathrm{dl}$ ).

\subsubsection{Exclusion criteria}

The exclusion criteria were: 1) Endocrine disorders, 2) Physical impairment that blocks physical activity, 3) Previous education of lifestyle, 4) Absence of more than two sessions of training classes.

\subsection{Data collection, examinations, and laboratory}

After reviewing the participants' records of the aforementioned project, potential participants with at least one abnormality in their lipid profiles who were eligible for the other criteria, were extracted. Then they were invited to attend the cardiovascular diseases research center along with their parents. The study objectives were explained, and they were examined in terms of exclusion criteria. Informed consent was obtained when they felt free to participate in the study. The data gathering tools in this study included a checklist of demographic characteristics and blood samples for the tests. Physical examinations were conducted under the supervision of a pediatrician by a pediatric resident. Laboratory tests were taken from the participants at the reference laboratory of Birjand University of Medical Sciences after 12 hours fasting and physical inactivity. Blood samples to measure fasting blood sugar (FBS), triglyceride (TG), high-density lipoprotein (HDL), low-density lipoprotein (LDL) and cholestrol (chol) were taken with a syringe $5 \mathrm{ml}$ (Bacton Dickinson) and evacuated in the tube containing separator gel and clot activator. They were held vertically until they clotted, and then the samples were centrifuged for 15 minutes with sigma centrifuge at RPM 3000. In less than an hour, laboratory tests were performed by an enzymatic method using Pars test kits and qualitative control by the Prestige 24i biochemical auto analyzer (Roche, Germany).

\subsection{Intervention}

The children and adolescents with dyslipidemia were given six 1- hour training sessions for six consecutive weeks (one session per week). The training was conducted at the cardiovascular Diseases Research Center of Birjand University of Medical Sciences, Iran, from 10am to $11 \mathrm{am}$.

\subsubsection{Content}

The content of the trainings were as follows: definition of blood lipids, saturated and unsaturated fats, normal amounts of blood lipids, diagnosis of hyperlipidemia, healthy lifestyle, physical activity and healthy diet, appropriate to the age of children and adolescents.

\subsubsection{Sessions}

The training sessions were as follows:

1) First Session: explaining the purpose of study, normal level of blood lipids and effect of fats on health, obesity and its complications.

2) Second Session: the role of exercise on blood lipids and health

3) Third Session: nutrition, healthy foods and the importance of eating breakfast on health

4) Fourth Session: effects of snacks consumption on health and replacing harmful snacks with healthy ones

5) Fifth Session: the role of sleep and rest on health

6) Sixth Session: reviewing the contents and summing up

\subsubsection{Instruments}

The tutorial was presented using the lecture method and the use of slides, and at the beginning of each session, we recalled the contents of the previous meeting. Moreover, colorful pamphlets were given to the participants to study.

\subsection{Follow up and communications}

Questions and possible ambiguities of the participants were answered over the phone. Six months after the last training session, subjects were rechecked for blood lipids and blood pressure in the same conditions as previous trials. Then, the results were compared.

\subsection{Statistical analyses and confounding variables}

The collected data were analyzed with descriptive statistics and analytical tests using SPSS v.16 (SPSS Inc., Chicago, Illinois, USA). Normality of data was checked with Kolmogorov-Smirnov test, and data analysis was 
carried out with Paired-samples t-test, Pearson correlation coefficient, and Independent-samples t-test. P-values of less than 0.05 were considered significant. Confounding variables such as economic status of the family or child perception of received education were controlled by selecting similar economic conditions and natural or intermediate IQs (85-115).

\subsection{Research ethics}

This study was conducted by the approval of the ethics committee of Birjand University of Medical Sciences (Ref: IR.BUMS.1394.8), which was gained by disclosing the research method and objectives, and after obtaining informed consent from all participants. In order to observe the ethics of research and to ensure that no harm occurred, if the participants needed to initiate drug therapy due to the impairments in all lipid profiles, they were not entered into the study and were given medical treatment. This research is accordant with ethical codes in vulnerable groups such as informed consent despite the presence of the decision maker (parents), answering parenting and child questions and considering the proportion of benefits to the detriment of the study.

\section{Results}

This study was performed on 50 children with dyslipidemia. A total of $52 \%$ of participants were female and $48 \%$ were male. The mean age of the participants was $12.77 \pm 1.84$ (9.5-16.5 years). The results of paired-samples t-test showed a significant difference between the mean of cholesterol (from 197.88 \pm 21.38 to $184.72 \pm 23.34, \mathrm{p}<0.001$ ) and low-density lipoprotein, LDL (from $118.86 \pm 22.53$ to $108.26 \pm 22.48, \mathrm{p}=0.008$ ) as a result of lifestyle modification before and after the study. There was no significant difference between the mean of fasting blood sugar, FBS $(\mathrm{p}=0.478)$, triglyceride, TG $(\mathrm{p}=0.721)$ and high-density lipoprotein, HDL $(\mathrm{p}=0.905)$ before and after the study (Table 1). The results of Independent-samples t-test showed no significant difference as a result of gender-based lifestyle modification between the two sexes in the mean of fasting blood sugar, FBS $(\mathrm{p}=0.120)$, total cholesterol, Cho $(\mathrm{p}=0.324)$, triglyceride, TG $(\mathrm{p}=0.713)$, high-density lipoprotein, HDL $(\mathrm{p}=0.540)$ and low-density lipoprotein, LDL $(p=0.430)$. However, levels of cholesterol and LDL in boys compared to girls were better improved after the study (Table 2). Pearson correlation coefficient showed no significant relationship between the mean of FBS $(\mathrm{r}=0.069, \mathrm{p}=0.634)$, Cho $(\mathrm{r}=-0.005, \mathrm{p}=0.973)$, TG $(\mathrm{r}=0.129, \mathrm{p}=0.374)$, HDL $(\mathrm{r}=0.053, \mathrm{p}=0.713)$ and LDL $(\mathrm{r}=$ $0.051, \mathrm{p}=0.724)$ in participants as a result of lifestyle modification and age (Table 3 ).

Table 1. Comparison of the mean of fasting blood sugar, total cholesterol, triglyceride, high-density lipoprotein and low-density lipoprotein in children before and after lifestyle modification

\begin{tabular}{|l|l|l|l|l|l|}
\hline Variables & Before $($ Mean \pm SD $)$ & After $($ Mean \pm SD $)$ & \multicolumn{3}{|l|}{ Paired-samples t-test } \\
\cline { 4 - 6 } & & & t-statistics & df & p-value \\
\hline FBS & $89.68 \pm 10.05$ & $88.24 \pm 8.94$ & 0.715 & 49 & 0.478 \\
\hline Cholesterol & $197.88 \pm 21.38$ & $184.72 \pm 23.34$ & 4.298 & 49 & $<0.001$ \\
\hline TG & $103.68 \pm 35.01$ & $103.68 \pm 35.01$ & 0.359 & 49 & 0.721 \\
\hline HDL & $52.02 \pm 12.42$ & $52.02 \pm 12.42$ & -0.120 & 49 & 0.905 \\
\hline LDL & $118.86 \pm 22.53$ & $108.26 \pm 22.48$ & 2.762 & 49 & 0.008 \\
\hline
\end{tabular}

Table 2. Comparison the mean of fasting blood sugar, total cholesterol, triglyceride, high density lipoprotein and low density lipoprotein in children before and after lifestyle modification based on gender

\begin{tabular}{|c|c|c|c|}
\hline \multirow{2}{*}{\multicolumn{2}{|c|}{ Variables }} & \multicolumn{2}{|l|}{$\mathrm{p}$-value } \\
\hline & & Paired-samples t-test* & Independent-samples t-test** \\
\hline \multirow[t]{2}{*}{ FBS } & Male & 0.120 & \multirow[t]{2}{*}{0.120} \\
\hline & Female & 0.565 & \\
\hline \multirow[t]{2}{*}{ Cholesterol } & Male & 0.001 & \multirow[t]{2}{*}{0.324} \\
\hline & Female & 0.024 & \\
\hline \multirow[t]{2}{*}{ TG } & Male & 0.524 & \multirow[t]{2}{*}{0.713} \\
\hline & Female & 1.000 & \\
\hline \multirow[t]{2}{*}{ HDL } & Male & 0.719 & \multirow[t]{2}{*}{0.540} \\
\hline & Female & 0.614 & \\
\hline \multirow[t]{2}{*}{ LDL } & Male & 0.033 & \multirow[t]{2}{*}{0.430} \\
\hline & Female & 0.127 & \\
\hline
\end{tabular}

* Comparing within groups (before and after), ${ }^{* *}$ Comparing between male and female 
Table 3. The Relationship between the mean of fasting blood sugar, total cholesterol, triglyceride, high-density lipoprotein and low-density lipoprotein in children as a result of lifestyle modification with age

\begin{tabular}{|l|l|l|}
\hline \multirow{2}{*}{ Variables } & \multicolumn{2}{|l|}{ Pearson's Correlation Coefficient Index } \\
\cline { 2 - 3 } & r & p-value \\
\hline FBS & 0.069 & 0.634 \\
\hline Cholesterol & -0.005 & 0.973 \\
\hline TG & 0.129 & 0.374 \\
\hline HDL & 0.053 & 0.713 \\
\hline LDL & -0.051 & 0.724 \\
\hline
\end{tabular}

\section{Discussion}

The study was conducted to evaluate the effect of lifestyle modification on serum lipids of children and adolescents with dyslipidemia. The results showed that lifestyle modification (physical activity and nutritional reform) is effective in reducing the level of cholesterol and harmful blood lipids, including LDL. The results showed a significant difference between the mean of cholesterol and low-density lipoprotein (LDL) as a result of lifestyle modification before and after the study. There was no significant difference between the mean of fasting blood sugar (FBS), triglyceride (TG), and high-density lipoprotein (HDL), before and after the study. A study by Kelishadi et al. that examined the impact of lifestyle modification includes avoiding fast foods and high-calorie foods, having breakfast, and increasing physical activity at home and school on body mass index (BMI) and lipid profiles of obese children and adolescents. The results of the study showed that lifestyle modification can be effective in reducing the blood level of LDL and total cholesterol (26). The results of that study are consistent with our study in terms of reducing LDL and cholesterol as a result of lifestyle modification. But in the study of Kelishadi et al., an increase in the level of HDL and TG reduction have been reported along with low-density lipoprotein (LDL) and cholesterol (Cho) reduction, which is not significant in our study. This difference can be justified by considering the sample size in the two studies, which consisted of 50 participants in this study and 412 in the study of Kelishadi et.al. Obviously, the greater sample size, the more likelihood of significant changes. Contrary to the past studies in which long-term educational interventions have been considered essential for reducing the lipid profiles (27, 28), the present study showed that 6 training sessions can also be effective in reducing the level of lipid profiles. Limitation of the educational sessions was reported in the study of Kelishadi et al. and only one educational session had been reported beneficial on lipid profiles of participants (26). Several studies have focused on the role of education on physical activity $(29,30,32)$, nutritional reform $(33,34)$, sleep and rest (35), and subsequently, lipid profiles, which are consistent with our study of the importance of education in reducing the lipid profiles through lifestyle modification.

Based on the study, no significant difference was found as a result of gender-based lifestyle modification between the two sexes. However, better changes at the level of two components (LDL, Cho) were seen in boys compared to girls after the study. Since this issue has not been reported in similar studies, we cannot really comment on it, but the impact of gender on the level of training effectiveness does not appear to be significant. The results showed no significant relationship between lifestyle modification and the age of participants in the mean of FBS, Cho, TG, HDL and LDL. Some studies confirm these results $(36,37)$ and others suggest patient's age at the time of modification is as an important factor on blood lipid levels $(29,38)$. However, it is suggested that this issue be investigated in a higher sample size study in order to obtain results that are more reliable.

\section{Conclusions}

Since inappropriate lifestyle, including physical inactivity and high calorie intake, is one of the most important causes of dyslipidemia in children and adolescents, according to the results of the study, lifestyle modification relying on exercise and physical activity, nutrition and sufficient sleep and rest can be effective as a nonpharmacological supplement in reducing blood lipids and dyslipidemia. The limitations of the study were the role of environmental factors such as school and peer group and lack of family cooperation.

\section{Acknowledgments:}

This paper is part of a pediatrics residency dissertation approved by Birjand University of Medical Sciences (Grant Number 743, November 2015). The authors would like to thank the Research Deputy of Birjand University of Medical Sciences, Clinical Research Center at Birjand University of Medical Sciences and all participants, without whom this study would not have been possible. 


\section{Conflict of Interest:}

There is no conflict of interest to be declared.

\section{Authors' contributions:}

All authors contributed to this project and article equally. All authors read and approved the final manuscript.

\section{References:}

1) Dholpuria R, Raja S, Gupta BK, Chahar CK, Panwar RB, Gupta R, et al. Atherosclerotic risk factors in adolescents. Indian J Pediatr. 2007; 74(9): 823-6. PMID: 17901667.

2) Antman EM, Selwyn AP, Braunwald E. Ischemic heart disease. In: Fauci AS, Braunwald E, Kasper D, Longo L, Hauser S L, Jameson J. L, Lascalza, J (editors). Harrison's Principle of Internal Medicine. 17th ed. New York, NY: McGraw-Hill. 2008; 1514-20.

3) Couch SC, Cross AT, Kida K, Ros E, Plaza I, Shea S, et al. Rapid westernization of children's blood cholesterol in 3 countries: evidence for nutrient- gene interactions? Am J Clin Nutr. 2000; 72 (suppl 5): 1266s-74s. doi: 10.1093/ajen/72.5.1266s. PMID: 11063468.

4) Barquera S, Pedroza-Tobias A, Medina C, Hernandez-Barrera L, Bibbins-Domingo K, Lozano R, et al. Global Overview of the epidemiology of Atherosclerotic Cardiovascular Disease. Archives of Medical Research. 2015; 46(5): 328-38. doi: 10.1016/j.arcmed.2015.06.006. PMID: 26135634.

5) Talaei M, Sarrafzadegan N, Sadeghi M, Oveisgharan SH, Marshal T, Thomas G N, Iranipour R. Incidence of Cardiovascular Diseases in an Iranian Population: The Isfahan Cohort Study. Arch Iran Med. 2013; 16(3): 138-44. doi: 013163/AIM.004. PMID: 23432164.

6) Ganz P, Ganz W. Coronary blood flow and mycardial ischemia. In: Braunwald E, Zipes DP, Libby P (eds). Braunwald's Heart Disease: A Textbook of Cardiovascular Medicine. 6th ed. Philadelphia; WB Saunders. 2001; 1105-6.

7) Daniels SR, Greer FR, Bhatia JJ, Schneider MB, Silverstein J, Stettler N, et al. Lipid screening and cardiovascular health in childhood. Pediatrics. 2008; 122(1): 198-208. doi: 10.1542/peds.2008-1349. PMID: 18596007.

8) Zapata LB, Bryant CA, McDermott RJ, Hcfelfinger JA. Dietary and physical activity behaviors of middle school youth: the youth physical activity and nutrition survey. J Sch Health. 2008; 78(1): 9-18; quiz 65-7. doi: 10.1111/j.1746-1561.2007.00260.x. PMID: 18177295.

9) Rey-Lopez JP, Vicente-Rodriguez G, Biosca M, Moreno LA. Sedentary behaviour and obesity development in children and adolescents. Nutr Metab Cardiovasc Dis. 2008; 18(3): 242-51. doi: 10.1016/j.numecd.2007. 07.008. PMID: 18083016.

10) Guedes D, Guedes J, Barbosa D, Oliveira, JD. Niveis de pratica de atividade fisica habitual em adolescentes. Rev Bras Med Esporte. 2001; 7(6): 187-99. doi: 10.1590/S1517-86922001000600002.

11) Bellize M.C, Graham W, Horgan F, Guillaume M, William H. Dietz. Prevalence of childhood and adolescent overweight and obesity in Asian and European countries. In: Chunming Chen, Dietz W.H (edt). Obesity in childhood and adolescence. Lippincott Williams \& Wilkins, Philadelphia, 2002; 23-32.

12) Skybo TA, Ryan- Wenger N. A school-based intervention to teach third grade children about the prevention of heart disease. Pediat Nurs. 2002; 28(3): 223-9, 235. PMID: 1208764.

13) Rackley CE, Schlant RC. Dyslipidiosis, other risk factor and prevention of coronary artery disease. In: Wayne RA, Schlant RC (eds). The Textbook of Heart Disease. 9th ed. New York; McGrow-Hill. 1997; Pp: 1213-21.

14) Radomyska B. Screening programme for hyper lipidemia in children and adolescents. Prophylactic -aspects of atherosclerosis. Med Wieku Rozwoj. 2001; 5(1): 27-34. PMID: 11276501.

15) Kelishadi R. Childhood overweight, obesity and the metabolic syndrome in Developing countries. Epidemiol Rev. 2007; 29: 62-76. doi: 10.1093/epirev/mxm003. PMID: 17478440.

16) Klishadi R, HashemiPour M, Zadegan NS, Kahbazi M, Sadry G, Amani A, et al. Dietary fat intake and lipid profiles of Iranian adolescents: Isfahan healthy heart program-heart health promotion from childhood. Prev Med. 2004; 39(4): 760-66. doi: 10.12691/jfnr-2-6-10. PMID: 15351543.

17) Azizi F, Rahmani M, Madjid M, Allahverdian S, Ghanbili J, Ghanbarian A, et al. Serum lipid levels in an Iranian population of children and adolescents: Tehran lipid and glucose study. Eur J Epidemiol. 2001; 17(3): 281-88. PMID: 11680549.

18) Dholpuria R, Raja S, Gupta BK, Chahar CK, Panwar RB, Gupta R, et al. Atherosclerotic risk factors in adolescents. Indian J Pediatr. 2007; 74(9): 823-6. PMID: 17901667. 
19) Antal M, Regoly-Merei A, Mesko E, Nagy K M, Barna M, Biro L, et al. Incidence of risk factors in parents with acute myocardial infarction at young age and in their children. Orv Hetil. 2004; 145(49): $2477-83$. PMID: 15633735.

20) Oksan D, Turner L, Hasanoglu A. Cholesterol screening in school children: is family history reliable to choose the ones to screen. Acta Pediatrica. 2007; 96(12): 1794-8. doi: 10.1111/j.1651-2227.2007.00554.x.

21) Robert M, Kligman B, Staton MD, Joseph ST. Disorders of lipoprotein metabolism and transport. $191 \mathrm{~h}$. united states. Sunders Ni. 2011: 80-88.

22) Austin MA, Hutter CM, Zimmem RL, Humphries SE. Familial hypercholesterolemia and coronary heart disease: a huge association review. Am J Epidemiol. 2004; 160(5): 421-429. doi: 10.1093/aje/kwh237. PMID: 15321838 .

23) Bhatnagar D, Soran H, Durrington PN. Hypercholesterolaemia and its management. BMJ 2008; $337: 503$ 8. doi: 10.1136/bmj.a993. PMID: 18719012.

24) Brunzell JD, William R. Hypertriglyceridemia in children. N Engl J Med. 2007; 357: 1009-17. doi: 10.1056/NEJMcp070061.

25) Jalali F, Haji Ahmadi M, Hosseimpour M, Angari MZ, Asadi E. Knowledge, attitude and practice (KAP) of people living in Babol about clinical symptoms and risk factors of coronary artery diseases (CAD). Journal of Babol University of Medical Sciences. 2004; 6(1): 43-9. In Persian.

26) Kelishadi R, Hashemipour M, Sheikh-Heidar A, Ghatreh-Samani S H. Changes in Serum lipid profile of obese or overweight children and adolescents following a lifestyle modification course. ARYA Atherosclerosis Journal. 2012, 8(3): 143-7. In Persian. PMID: 23359278, PMCID: PMC3557010.

27) Knip M, Nuufinen Q. Long- term effects of weight reduction on serum lipids and plasma insulin in 0bese children. Am J Clin Nutr. 1993; 57(4): 490-3. doi: 10.1093/ajcn/57.4.490.

28) Wadden TA, Anderson DA, Foster GD. Two-year changes in lipids and lipoproteins associated with the maintenance of a $5 \%$ to $10 \%$ reduction in initial weight: some findings and some questions. Obes Res. 1999; 7(2): 170-8. doi: 10.1002/j.1550-8528.1999.tb00699.x. PMID: 10102254.

29) Lim JS. The current state of dyslipidemia in Korean children and adolescents and its management in clinical practice. Ann Pediatr Endocrinol Metab. 2013; 18(1): 1-8. doi: 10.6065/apem.2013.18.1.1. PMID: 24904843, PMCID: PMC4027065.

30) Avila- Rodriguez A, Avila- Rodriguez EH, Araujo- Contreras JM, Rivas-Avila E, Lee RE, RodriguezOrtega LJ, et al. Relationship between Dyslipidemia and Physical Activity in Mexican Children. International Journal of Exercise science. 2012; 6(2).

31) Zardast M, Namakin, K, Chahkandi T, Taheri F, Kazemi T, Bijari B. Prevalence of Metabolic Syndrome in Elementary School Children in East of Iran. J Cardiovasc Thorac Res. 2015; 7(4): 158-163. doi: 10.15171/jcvtr.2015.34. PMID: 26702345, PMCID: PMC4685282.

32) Rômulo Araújo Fernandes, Diego Giulliano Destro Christofaro, Juliano Casonatto, Jamile Sanches Codogno, Eduardo Quieroti Rodrigues, Mauro Leandro Cardoso, et al. Prevalence of dyslipidemia in individuals physically active during childhood, adolescence and adult age. Arq Bras Cardiol. 2011; 97(4): 317-23. doi: 10.1590/S0066-782X2011005000083. PMID: 21830000.

33) Bazzano LA, Serdula MK, Liu S. Dietary intake of fruits and vegetables and risk of cardiovascular disease. Curr Atheroscler Rep. 2003; 5(6): 492-9. PMID: 14525683.

34) de Alcantara Neto OD, Silva Rde C, Assis AM, Pinto Ede J. Factors associated with dyslipidemia in children and adolescents enrolled in public schools of Salvador Bahia, Alcantara Neto. Rev Bras Epidemiol. 2012; 15(2): 335-45. PMID: 22782099.

35) Basta M, Chrousos GP, Vela-Bueno A, Vgontzas An. Chronic Insomnia and Stress System. Sleep Med Clin. 2007; 2(2): 279-291. doi: 10.1016/j.jsmc.2007.04.002. PMID: 18071579, PMCID: PMC2128619.

36) Silverstein J, Haller M. Coronary artery disease in youth: present markers, future hope? J Pediatr. 2010; 157( 4): 523-4. doi: 10.1016/j.jpeds.2010.07.010. PMID: 20728091.

37) Ramezani M, Azizi F. Prevalence of Hyperlipidemia in 3-19 years old children and adolescent of Tehran in 2000. Iranian Journal of Endocrinology and Metabolism. 2005; 7(3): 249-53.

38) Ribas SA, Santana da Silva LC. Anthropometric indices: predictors of dyslipidemia in children and adolescents from north of Brazil. Nutr Hosp. 2012; 27(4): 1228-35. doi: 10.3305/nh.2012.27.4.5798. PMID: 23165566. 